\title{
Correcting and interpreting the effect of cognitive therapy versus exposure in anxiety disorders
}

\author{
Shanil Ebrahim ${ }^{1 *}$ and Sheena Bance ${ }^{2}$
}

\begin{abstract}
Dr. Ougrin's evaluation of cognitive therapy versus exposure in anxiety disorders reported a standardised mean difference [SMD] $(95 \%$ confidence interval $[\mathrm{CI}])$ of $0.52(0.37,0.74)$ for short-term outcomes and $0.46(0.29,0.73)$ for long-term outcomes in social phobia, and $0.88(0.69,1.11)$ for short-term outcomes and $1.05(0.80,1.37)$ for long-term outcomes in posttraumatic stress disorder (PTSD). These were incorrectly meta-analysed. Upon re-analysis, we found that the correct SMD $(95 \%$ Cl) was $-0.66(-1.19,-0.14)$ for short-term outcomes and mean difference $(95 \% \mathrm{Cl})$ of $-29.66(-46.13,-13.19)$ on the Social Phobia subscale from the Social Phobia Anxiety Inventory for long-term outcomes in Social Phobia. For PTSD, the SMD (95\% Cl) for short-term outcomes was -0.13 $(-0.36,0.11)$ and $0.05(-0.22,0.32)$ for long-term outcomes. However, correcting the errors did not change the interpretation of the findings considerably.
\end{abstract}

Keywords: Cognitive therapy, Exposure, Anxiety disorders, Systematic review, Meta-analysis, Correspondence, Correction

We read with great interest the systematic review evaluating the efficacy of exposure versus cognitive therapy (CT) in anxiety disorders, performed by Dr. Dennis Ougrin [1]. The author meta-analysed 20 randomised controlled trials (RCTs) comparing CT versus exposure in four anxiety disorders and concluded the following: "there appears to be no evidence of differential efficacy between cognitive therapy and exposure in PD [panic disorder], PTSD [posttraumatic stress disorder] and OCD [obsessive compulsive disorder] and strong evidence of superior efficacy of cognitive therapy in social phobia" [1].

Upon reviewing the short-term and long-term results of CT versus exposure in patients with Social Phobia, we found two errors. First, the summary effect (standard error [SE]) for the short-term outcome in Hofmann (2004) was incorrectly inputted as $-0.28(0.26)$. We contacted Dr. Stefan Hofmann [2], obtained the raw data from the published trial, and found that the correct summary effect (SE) was -0.19 (0.27). Second, Dr.

\footnotetext{
* Correspondence: shanil.ebrahim@utoronto.ca

${ }^{1}$ Department of Clinical Epidemiology and Biostatistics, McMaster University, 1200 Main Street West, Hamilton, Ontario, Canada

Full list of author information is available at the end of the article
}

Ougrin states, "the overall effect (the end-of-treatment standardised mean difference (SMD), Hedge's g) is summarised in Figure 7" [1]. The forest plot, however, does not indicate what summary effect was reported in the pooled analysis and thus the assumption was made that it was the SMD. Upon replicating the meta-analysis, we found that the summary effect was incorrectly pooled as an odds ratio (OR), i.e. OR (95\% confidence interval $[\mathrm{CI}])$ of $0.52(0.37,0.74)$ for short-term outcomes. Thus, we inputted the correct summary effect for Hofmann 2004 and re-analysed the meta-analysis using SMD for our summary effect, as originally intended. We found that the corrected SMD (95\% CI) was -0.66 (-1.19, -0.14) for short-term outcomes. We used the random effects model given that the $\mathrm{i}^{2}$ was $56 \%$ (Figure 1). Using the Cohen's d criteria of 0.2 to represent a small effect, 0.5 a medium effect and 0.8 a large effect [3], we found that CT has a medium effect in improving social phobia versus exposure with the lower bounds of the $95 \% \mathrm{CI}$ in the range of a modest effect, for short-term outcomes.

Long-term outcomes for social phobia were also incorrectly pooled as an OR. We found that the corrected SMD $(95 \%$ CI $)$ was $0.46(0.29,0.73)$ for long-term

\section{Biomed Central}




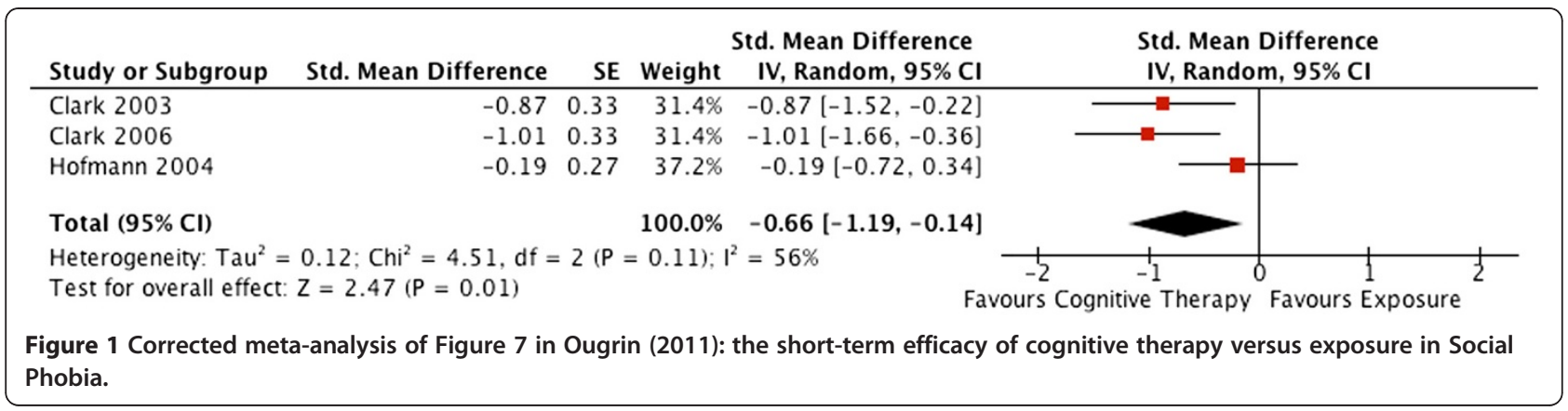

outcomes. However, the SMD should only be used when pooling different assessments for the same outcome. If the same assessment is reported across trials, the mean difference (MD) is always preferred. Both Clark (2006) and Hofmann (2004) [2,4] reported social phobia subscale scores from the Social Phobia and Anxiety Inventory (SPAI) [5]. Thus, we calculated the MD (95\% CI) as -29.66 (-46.13, -13.19) (Figure 2).

\section{Additional errors identified by Professor Julio Sánchez-Meca}

Professor Julio Sánchez-Meca, who was a reviewer of our article, identified additional errors in Dr. Ougrin's paper as follows:

1. In Figure 3 in Ougrin (2011), the forest plot was incorrectly pooled as odds ratios when the effect sizes were in fact SMDs (see Table 2 in Ougrin). We found that the corrected SMD (95\% CI) was -0.13 $(-0.36,0.11)$ for short-term outcomes in PTSD (Figure 3).

2. Figure 4 in Ougrin (2011) presents the same error as above. We found that the corrected SMD (95\% CI) was $0.05(-0.22,0.32)$ for long-term outcomes in PTSD (Figure 4).

3. On page 4 in Ougrin (2011), second column, fifth paragraph, reporting the results of the meta-analysis for short-term outcomes in panic disorder, an $\mathrm{I}^{2}$ of $68 \%$ is reported. However, in Figure 5 in Ougrin (2011), an $\mathrm{I}^{2}$ of $62 \%$ is reported.

4. On page 4 in Ougrin (2011), second column, last paragraph, reporting the results of the meta-analysis for long-term outcomes in panic disorder, an $\mathrm{I}^{2}$ of $24 \%$ is reported. However, in Figure 6 in Ougrin (2011), an $\mathrm{I}^{2}$ of $69 \%$ is reported. In addition, the author reported that a fixed effects model was applied when actually, a random-effects model was employed.

\section{Conclusions}

In summary, after correcting and re-analysing the metaanalyses, we found a medium effect in CT improving social phobia in comparison to exposure. It is important to note, however, that correcting the errors did not change the interpretation of the findings considerably in Dr. Ougrin's paper. Our paper stresses the importance of critically checking one's data and analyses to ensure validity of results.

\section{Response}

By Dennis Ougrin dennis.ougrin@kcl.ac.uk

I would like to thank Ebrahim and Bance for confirming and essentially independently replicating the main results of the meta analysis evaluating the efficacy of

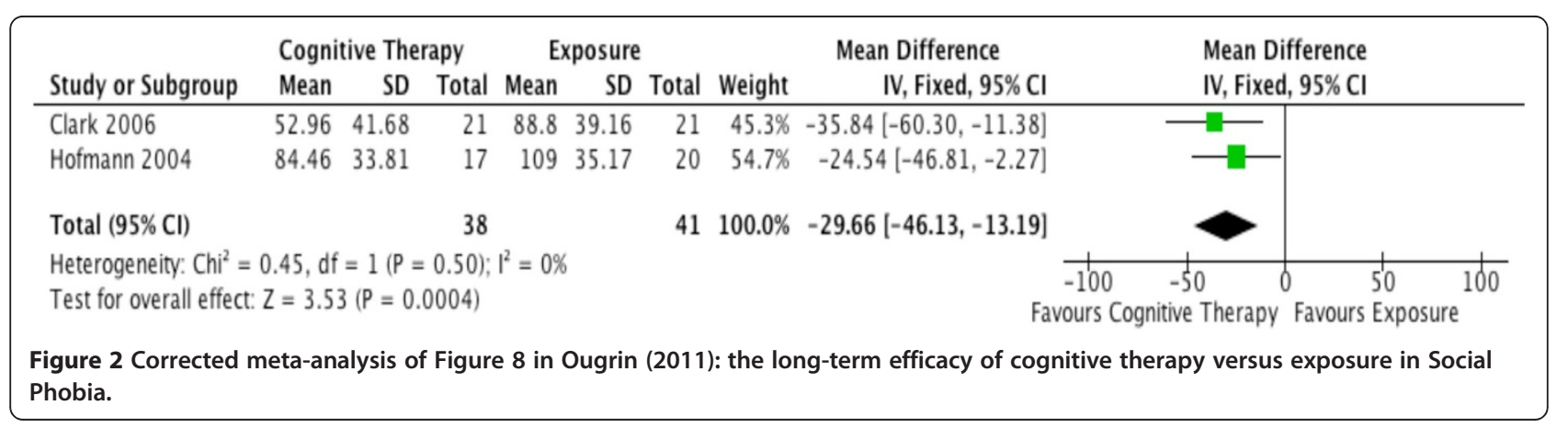




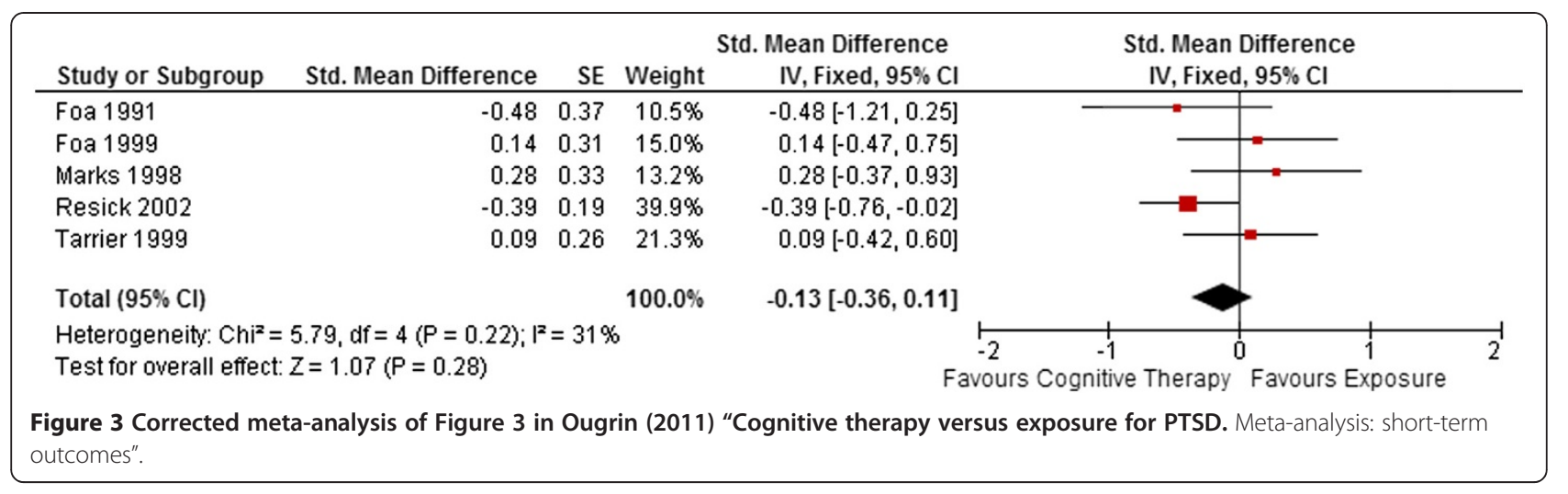

exposure versus cognitive therapy $(\mathrm{CT})$ in anxiety disorders [5]. Ebrahim and Bance reviewed the short-term and long-term results of CT versus exposure in patients with social phobia and found a slightly different standardised mean difference (SMD) and standard error [SE] for the short-term outcomes in Hofmann (2004) [1]: $-0.19(0.27)$ rather then $-0.28(0.26)$. Ebrahim and Bance did not state how their calculation was made. Since different formulae exist for this calculation it might be helpful if the authors provide the entirety of their calculations rather then only providing the final figure. The authors further used their calculation of the SMD in Hofmann (2004) [1] to re-calculate the pooled SMD, using random effects model instead of the original fixed effects model (due to the increased heterogeneity) and found the pooled SMD to be $-0.66(-1.19,-0.14)$, slightly greater then the original estimate but with wider confidence intervals. The recalculation of long-term outcomes for social phobia yielded essentially similar results. However, the authors substituted the SMD with the mean difference (MD) from the Social Phobia and Anxiety Inventory (SPAI) as the SPAI results were reported by all three studies used to calculate the pooled
SMD [1-6]. This may be a preferred strategy in most cases, however for the purpose of the original meta analysis and a priori decision was made to use the main outcome measure in each study. In both Clark (2003) and Clark (2006) the main outcome measure was a social anxiety composite, hence the decision was to use the SMD instead of the MD. Ebrahim and Bance's recalculation of the SMD in the PTSD [posttraumatic stress disorder] studies yielded essentially similar results as those in the original meta analysis. I would like to thank the authors for spotting several typos and some reporting inconsistencies which sadly even a very thorough peer review may miss. One important lesson is to check all default settings in the software used for meta analyses. Finally I would like to emphasise that confirmations and independent replications are extremely important and still quite rare in the field of psychological therapies. Both clinicians and researchers may feel more confident that extant literature does indeed indicate no overall difference between cognitive therapy (including behavioural experiments) and exposure for in PD [panic disorder], PTSD and OCD [obsessive compulsive disorder] and points to superior efficacy of cognitive therapy in social phobia.

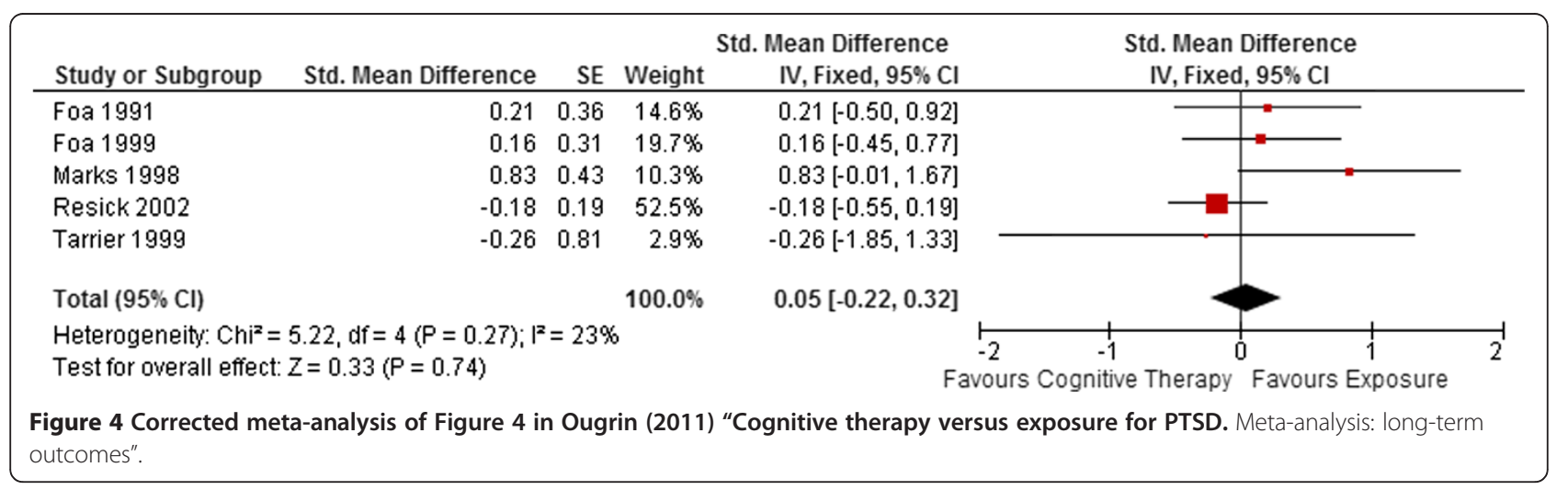




\section{Competing interests}

The authors declare that they have no competing interests.

\section{Authors' contributions}

SE and SB prepared, critically revised and approved the final manuscript to be published.

\section{Authors' information}

$\mathrm{SE}$ is a doctoral candidate in the Department of Clinical Epidemiology and Biostatistics at McMaster University in Hamilton, Ontario, Canada. SB is a master's student in the Department of Adult Education and Counselling Psychology at the University of Toronto in Toronto, Ontario, Canada.

\section{Acknowledgements}

We thank Professor Julio Sánchez-Meca, a reviewer of our article, who made important contributions in identifying additional errors in Dr. Ougrin's paper SE is supported by a Canadian Institutes of Health Research Doctoral Award.

\section{Author details}

'Department of Clinical Epidemiology and Biostatistics, McMaster University, 1200 Main Street West, Hamilton, Ontario, Canada. ${ }^{2}$ Department of Adult Education and Counselling Psychology, University of Toronto, Toronto, Canada.

Received: 20 March 2012 Accepted: 15 October 2012

Published: 20 November 2012

\section{References}

1. Ougrin D: Efficacy of exposure versus cognitive therapy in anxiety disorders: systematic review and meta-analysis. BMC Psychiatry 2011, 11(1):200

2. Hofmann S: Cognitive Mediation of Treatment Change in Social Phobia. J Consult Clin Psychol 2004, 72(3):392-399.

3. Cohen J: Statistical Power Analysis for the Behavioral Sciences. 2nd edition. Hillsdale, NJ: Lawrence Eribaum Associates; 1988. vol. 25.

4. Clark DM, Ehlers A, Hackmann A, McManus F, Fennell M, Grey N, Waddington L, Wild J: Cognitive therapy versus exposure and applied relaxation in social phobia: A randomized controlled trial. J Consult Clin Psychol 2006, 74(3):568-578.

5. Turner SM, Beidel DC, Dancu CV, Stanley MA: An empirically derived inventory to measure social fears and anxiety: The Social Phobia and Anxiety Inventory. Psychol Assess 1989, 1:35-40.

6. Clark DM, Ehlers A, McManus F, Hackmann A, Fennell M, Campbell H, Flower T, Davenport C, Louis B: Cognitive therapy versus fluoxetine in generalized social phobia: a randomized placebo-controlled trial. J Consult Clin Psychol 2003, 71(6):1058-1067.

doi:10.1186/1471-244X-12-202

Cite this article as: Ebrahim and Bance: Correcting and interpreting the effect of cognitive therapy versus exposure in anxiety disorders. BMC Psychiatry 2012 12:202.

\section{Submit your next manuscript to BioMed Central and take full advantage of:}

- Convenient online submission

- Thorough peer review

- No space constraints or color figure charges

- Immediate publication on acceptance

- Inclusion in PubMed, CAS, Scopus and Google Scholar

- Research which is freely available for redistribution 